\title{
Pratiques
}

Linguistique, littérature, didactique

\section{Le prédicat en question : objectifs d'enseignement, conception de la langue et choix d'une terminologie}

The predicate in question: teaching objectives, language design and choice of terminology

\section{Bernard Combettes}

\section{(2) OpenEdition}

Journals

Édition électronique

URL : http://journals.openedition.org/pratiques/3809

DOI : $10.4000 /$ pratiques.3809

ISSN : 2425-2042

Éditeur

Centre de recherche sur les médiations (CREM)

Référence électronique

Bernard Combettes, «Le prédicat en question : objectifs d'enseignement, conception de la langue et choix d'une terminologie », Pratiques [En ligne], 175-176 | 2017, mis en ligne le 22 décembre 2017, consulté le 21 avril 2019. URL : http://journals.openedition.org/pratiques/3809; DOI : 10.4000/ pratiques.3809

Ce document a été généré automatiquement le 21 avril 2019

(c) Tous droits réservés 


\title{
Le prédicat en question : objectifs d'enseignement, conception de la langue et choix d'une terminologie
}

The predicate in question: teaching objectives, language design and choice of terminology

\author{
Bernard Combettes
}

\section{Quelle terminologie pour quels objectifs d'enseignement?}

Les derniers Programmes d'enseignement de novembre 2015 proposent quelques changements en ce qui concerne la terminologie grammaticale. La discussion sur le bienfondé de ces modifications ne doit pas masquer la nature du vrai débat. La question de la nomenclature ne peut être traitée que dans le cadre général des objectifs visés et la justification des changements devrait avant tout s'appuyer sur des arguments montrant la cohérence avec le type d'enseignement proposé. Le souci de "simplification", d'homogénéisation, s'il est tout à fait compréhensible, ne peut suffire à lui seul, à motiver des modifications. Sans une mise en perspective plus large, toutes les hypothèses sont possibles : pourquoi ne pas penser, à la limite, qu'il s'agit de simplifier pour rendre plus « efficace » la démarche traditionnelle, ce qui ne serait qu'une façon de ne rien changer? Le texte officiel présente en fait des points de vue novateurs et des propositions particulièrement intéressantes qui, pour la première fois, sont exprimées aussi explicitement. C'est donc par rapport à ces prises de position que doit être justifié le changement terminologique. On ne voit pas très bien en effet quel serait le « bénéfice » de l'introduction de nouvelles notions, comme prédicat ou complément de verbe, s'il s'agissait simplement de remplacer des étiquettes déjà bien entrées dans l'usage et de continuer en même temps des activités de reconnaissance, d'identification, destinées à faire acquérir les notions grammaticales renvoyant essentiellement à des natures et à des fonctions, 
sans que rien ne soit fondamentalement modifié dans les pratiques. L'actualisation de la nomenclature a déjà eu lieu, de façon plus ou moins profonde, - on peut citer, en particulier, la «Terminologie grammaticale» de 1997, - au fil des textes officiels depuis bon nombre d'années ; or un rapide examen des manuels suffit pour constater qu'aucun changement majeur n'est intervenu dans les activités proposées, qui consistent essentiellement à faire identifier une catégorie ou une fonction.

Quelle articulation des principes généraux et des pratiques les nouveaux textes proposent-ils ? Y a-t-il cohérence entre les choix opérés en matière de terminologie et les démarches préconisées? C'est ce que nous voudrions rapidement examiner, avant de porter un jugement sur les innovations proposées.

\section{Observer le système de la langue}

3 En matière d'enseignement de la langue, l'ensemble du dispositif, tant en ce qui concerne les contenus que les démarches, devrait découler d'une prise de position que l'on ne peut qu'approuver : un des objectifs principaux est l'observation du système de la langue, la réflexion sur les structures linguistiques. Le texte présente en effet en plusieurs endroits des formulations qui rappellent l'importance du « raisonnement linguistique », comme dans ce passage, qui figure sous le titre : «La grammaire au service de la réflexion sur la langue » :

L'objectif n'est pas la mémorisation de règles ou d'étiquettes grammaticales pour elles-mêmes, mais bien la formation intellectuelle des élèves et le développement d'une posture réflexive. Il s'agit de leur faire percevoir que la langue est un système organisé et de les faire réfléchir sur les normes, la pertinence et l'acceptabilité de telle ou telle forme [...] (MENESR, 2015, p. 236)

4 À travers ce type de proposition se trouve nettement affirmée la nécessité d'observer le fonctionnement du système linguistique, cette observation n'étant pas une fin en soi mais allant évidemment de pair avec l'acquisition, par le renforcement de la pratique, des diverses structures.

5 On peut sans doute regretter que cet objectif ne soit pas mieux mis en relief et n'apparaisse qu'en dernier lieu après des finalités d'un autre ordre, qui n'auraient pas dû être placées sur le même plan. La hiérarchisation des divers objectifs n'est sans doute pas d'une très grande clarté dans les nouveaux programmes, et on s'étonnera par exemple que soient séparés « le développement des compétences langagières orales et écrites en réception et en production » et « l'approfondissement des compétences linguistiques qui permettent une compréhension synthétique du système de la langue » (ibid., p. 227), alors que ces deux objectifs sont étroitement liés et que leur autonomisation fait ressurgir l'écueil de «la grammaire pour la grammaire ». Plutôt que cette présentation très dichotomique, des indications sur les façons d'articuler étude du système et compétences en production et en compréhension auraient mieux répondu aux idées de fond qui soustendent ces textes.

Ceci étant, le plus discutable - et le plus décevant - est le fossé qui sépare cet objectif d'observation du système de la langue et les moyens suggérés pour y parvenir, dans la mesure où ces moyens correspondent au maintien, quasiment à l'identique, de la priorité accordée à l'« analyse ", au sens restreint du terme, limitée à la décomposition et à l'identification. Quelles sont en effet les activités proposées, quelles sont les compétences et les connaissances que ces activités visent à mettre en place? Sous l'objectif général 
« connaitre les aspects fondamentaux du fonctionnement syntaxique ( (ibid., p. 237) sont par exemple énumérées les activités suivantes :

- analyse des constituants de la phrase simple

- identification des groupes : leurs constituants et leurs fonctions

- identification des classes de mots et mise en évidence de leurs propriétés

Ou encore, lorsqu'il s'agit d'aborder le « fonctionnement » de la phrase complexe :

- identification des constituants de la phrase

Il semble peu acceptable de ramener l'observation du système linguistique à la reconnaissance des unités; même modernisé dans ses contenus, même modifié dans certaines de ses notions, c'est bien le même type d'enseignement qui se maintient. Une recommandation comme: «Au cycle 4, [...] c'est dans la perspective de leur fonctionnement syntaxique que sont étudiées les classes de mots et leurs relations » (ibid., p. 236) a sans doute pour but d'éviter "l'analyse pour l'analyse", mais la démarche demeure toujours la même : ce sont les classes et les fonctions qui sont l'objet de l'étude, même si c'est dans une autre "perspective ", alors qu'il aurait fallu donner la priorité aux structures et à leur fonctionnement. La formulation adoptée laisse penser que l'on étudie d'abord natures et fonctions avant de passer à l'examen du fonctionnement syntaxique, ce qui est en contradiction avec les objectifs visés. Aucune amélioration ne sera possible s'il n'est pas indiqué, le plus clairement et le plus concrètement possible, ce que peut être l'observation - et l'explication - d'un fait de langue, d'une structure syntaxique particulière, étudiée pour elle-même et non comme prétexte à découvrir natures et fonctions. Seuls des exemples précis de la démarche à suivre donneront aux enseignants une idée, en décomposant l'énoncé en divers constituants dont on reconnaitra la nature, que seront observés le fonctionnement et les propriétés de telle ou telle construction, qu'il s'agisse, pour ne citer que quelques exemples, des structures clivées, des verbes « symétriques » (on casse la banche/la branche casse), des constructions factitives, ou encore des subordonnées relatives. Ceci ne signifie pas que l'identification des natures et des fonctions soit inutile, mais, outil pour l'observation et la réflexion, elle doit être justifiée par l'étude des phénomènes et ne peut constituer un objectif préalable.

Dans leur ouvrage fondateur, Linguistique et enseignement du français (1970), É. Genouvrier et J. Peytard avaient déjà signalé le risque d'un tel écart, et, en accord avec leur objectif général, proposaient une observation du système dépassant le simple étiquetage : «On remarquera qu'il ne s'agit pas d'étudier tel ou tel type de complément, mais le fonctionnement linguistique d'une structure considérée dans sa totalité » (ibid., p. 165). Et, plus loin: "Il ne s'agit en aucun cas de se contenter d'une analyse des fonctions au sens habituel du terme, mais d'étudier des procédures linguistiques » (ibid.)

En ce qui concerne la terminologie, les conséquences de cette réorientation vers l'observation $\mathrm{du}$ fonctionnement $\mathrm{du}$ système semblent évidentes: ce qui est indispensable, ce n'est pas tant une nomenclature concernant les unités qu'un état des structures de langue qu'il conviendra d'aborder avec les élèves. Cela reviendrait à renverser, en quelque sorte, les priorités, à mettre en avant les constructions, les relations syntaxiques, qui sont l'objet de l'observation, et non les unités qui entrent en jeu dans ces relations. Travailler sur les constructions du verbe, sur la valence, n'est pas du même ordre que faire reconnaitre le complément d'objet, qu'on l'appelle ainsi ou autrement ; travailler sur la construction impersonnelle, avec l'observation de toutes les contraintes et des propriétés qu'elle comporte, n'est pas du même ordre que faire 
reconnaitre un sujet ou un verbe à la forme impersonnelle. C'est un véritable programme de langue qui est attendu, et non un programme de grammaire, ce dernier ne pouvant que dépendre du programme de langue, aussi bien en ce qui concerne les notions que la progression. On regrettera donc que n'apparaissent pas des termes comme «valence verbale ", " verbes symétriques ", « constructions clivées », ... et, par ailleurs, que ne soit pas indiqué à quel niveau tel ou tel de ces faits de langue serait à étudier.

11 Se pose ici au passage une question qui devrait être prise en compte par des textes officiels: ne serait-il pas utile d'établir un ensemble de notions - et par là, une terminologie - destiné seulement aux enseignants, cette terminologie n'étant pas obligatoirement utilisée avec les élèves?

\section{Prendre en compte la relation syntaxe/sémantique}

12 Un autre aspect novateur, qui devrait avoir des relations étroites avec la terminologie, apparait également dans des nouveaux Programmes de 2015 ; il concerne la conception même de la syntaxe. S'ils n'ont pas pour but de prendre la place d'un manuel, les textes officiels ne peuvent éviter de renvoyer, plus ou moins explicitement, à des prises de position théoriques. Une description de la langue n'est pas neutre; liée à l'état de la science, elle s'appuie sur des points de vue concernant la nature et le fonctionnement du langage et de la langue. Alors que les textes antérieurs étaient peu explicites sur ce point, les nouveaux programmes font preuve d'un peu plus de clarté et de précision. Le texte officiel souligne en effet à plusieurs reprises la priorité des constructions sur les unités et insiste sur l'importance du lien qu'il convient d'établir entre la syntaxe et la sémantique ; il se démarque ainsi d'une conception de la "syntaxe autonome", conception qui était plus ou moins sous-jacente à la "grammaire structurale" fondée sur le distributionalisme. Ce double souci de privilégier les structures syntaxiques, fondement du système, et de ne pas les isoler des contenus qu'elles ont pour fonction de coder constitue incontestablement un point très positif, qui n'avait jamais été formulé aussi nettement. La notion de «construction » apparait ainsi fréquemment, en liaison avec le niveau sémantique. Par exemple, au cycle 3 (MENESR, 2015, p. 116) :

- Mise en évidence du lien sens-syntaxe : place et rôle du verbe, constructions verbales, compléments du verbe et groupe verbal.

- Comparaison de constructions d'un même verbe, catégorisation (rapport sens-syntaxe) et réemploi (jouer avec, jouer à, jouer pour... / la plante pousse - Lucie pousse Paul).

Ou encore, au cycle 4 (ibid., p. 240) :

- Identification des verbes à construction directe et à construction indirecte, des verbes à plusieurs compléments; mise en évidence des constructions par la pronominalisation; analyse du sens des verbes en fonction de leur construction.

- Identification des verbes pronominaux.

- Manipulations syntaxiques pour mettre en évidence les constructions verbales.

Il est cependant regrettable que les exemples choisis pour illustrer cette problématique laissent penser qu'elle ne concerne que les constructions du verbe, ce qui risque de conduire à une dérive vers des études essentiellement lexicales, alors que la problématique devrait intéresser l'ensemble du niveau syntaxique. L'observation du fonctionnement de la phrase passive, de la phrase impersonnelle, etc. doit prendre en compte l'interface syntaxe/sémantique et cela demande que soit introduites des notions qui permettent de caractériser les syntagmes qui entrent dans ces constructions, comme 
les traits abstrait/concret, animé/non animé, massif/comptable, etc. Ici encore, comme cela a été suggéré plus haut, une nomenclature réservée aux enseignants apparait comme indispensable, ce qui est proposé dans les rubriques «lexique» étant tout à fait insuffisant.

\section{Les modifications de la terminologie}

15 C'est dans cette double optique, celle d'un enseignement privilégiant l'observation du système et non l'analyse - au sens traditionnel de reconnaissance des unités -, celle d'une approche mettant l'accent sur l'interface syntaxe/sémantique, qu'il convient d'évaluer la pertinence des changements concernant la terminologie. Ceux-ci sont au nombre de quatre :

- l'introduction de la notion de " prédicat »;

- le remplacement des divers compléments d'objet par la notion de " complément du verbe »;

- la suppression de la liste, d'ordre sémantique, des compléments circonstanciels ;

- la suppression de la notion de "proposition principale ».

Ces quatre points ne sont pas du même ordre et ne relèvent pas tous du même niveau de discussion. Deux d'entre eux en effet peuvent être rattachés à la problématique qui vient d'être évoquée : la question du prédicat et celle de la proposition principale. Il s'agit de déterminer dans quelle mesure ces unités sont pertinentes dans une démarche d'observation des caractéristiques du système syntaxique. La question des compléments, en revanche, qu'il s'agisse des objets ou des circonstanciels, ne présente pas le même intérêt. Dans la mesure où l'accent est mis sur les constructions, il semble plus urgent de délimiter clairement et de nommer les diverses structures à prendre en compte, les « fonctions » ne constituant pas un objet d'étude prioritaire.

\section{La proposition principale}

La suppression de la notion de "proposition principale» apparait comme tout à fait justifiée dans le cadre général qui est tracé, tant au plan scientifique qu'au plan didactique. Elle a en quelque sorte pour but de proposer une même approche, là où la tradition distinguait deux types d'analyse, celle de la phrase simple et celle de la phrase complexe (l'analyse «logique»). Cette distinction concernait essentiellement le découpage en unités, les fonctions des subordonnées étant les mêmes (sujet, objet, attribut, etc.) que celles des constituants de la phrase simple. On remarquera d'ailleurs que la fonction d'une complétive était celle d'objet du verbe de la principale, et non d'objet de la principale, que la fonction de la relative était d'être complément de son antécédent et non d'être un complément de la principale, ce qui montre bien la situation en porte à faux de l'analyse logique. Les opérations de substitution, entrées dans la pratique depuis un certain temps, ont habitué à faire apparaitre des équivalences de fonctionnement entre les subordonnées et les constituants nominaux ou adjectivaux. Les subordonnées ont évidemment, dans bon nombre de cas, un comportement spécifique, mais cela ne justifie pas pour autant que l'on identifie une unité qui correspondrait à une « principale». Tout n'est cependant pas aussi simple; dans un certain nombre de cas, la subordonnée n'entretient pas de relation de dépendance avec un constituant particulier du contexte, mais avec l'ensemble de l'énoncé dans lequel elle est enchâssée, ce qui pourrait sembler justifier la dénomination de «principale ». C'est par exemple le cas de 
circonstancielles comme les temporelles antéposées: Quand il fait beau, $X$ va se promener, ou encore des relatives " appositives", comme dans: $X$ n'est pas venu, ce qui est étrange. Il serait de bonne méthode de continuer l'alignement sur la phrase simple, en mettant en parallèle ces constructions avec des tours comme : Aux beaux jours, $X$ va se promener, $X$ n'est pas venu, chose étrange. Les circonstants détachés sont souvent analysés comme des " compléments de phrase ", terme peu pertinent dans la mesure où ils font eux-mêmes bien partie de la phrase. La dénomination de " complément de proposition " - qui n'exige pas que l'on introduise le terme de " principale » - serait sans doute plus juste. Dans le cas de l'apposition, de la même façon qu'il y a des relatives apposées à des groupes nominaux, on pourrait parler d'une relative apposée à une proposition, ce qui n'appelle pas non plus le terme de principale.

\section{Les compléments}

Nous passerons rapidement sur la simplification qui concerne le traitement des compléments ; qu'il s'agisse des objets ou des circonstants, elle découle logiquement, elle aussi, du cadre général proposé. Puisqu'il est question de mettre l'accent sur les constructions, sur les structures syntaxiques, la notion de "complément de verbe" apparait en effet comme suffisante, l'essentiel étant l'observation des possibilités de construction offertes par telle ou telle forme verbale. Cette dénomination permet également de résoudre la question des compléments régis du type aller à $X$, être à tel endroit, etc., que l'on était réticent à nommer « objets du verbe ", l'emploi de l'étiquette « complément essentiel » réglant en partie la difficulté. Si la dénomination « complément de verbe" présente un certain avantage, il conviendrait surtout de proposer une nomenclature - peut-être à l'intention des enseignants, cela est à discuter - pour les constructions. Des expressions comme "voix passive", "construction impersonnelle", "forme pronominale ", sont quelque peu hétéroclites et sont loin de recouvrir l'ensemble des possibilités. Parlera-t-on par exemple de "verbes symétriques ", de "construction factitive » ? La clarification sur ces points est nécessaire, en particulier s'il s'agit de proposer des progressions.

\section{Le prédicat}

La notion de prédicat est celle qui a soulevé le plus de discussions et de critiques. Il a semblé en effet qu'il y avait là l'introduction d'un terme dont on voyait mal la pertinence, dans la mesure où il pouvait faire double emploi avec celui de groupe verbal, bien entré dans l'usage. Il est vrai que la plupart des arguments avancés pour justifier cette introduction peuvent s'appliquer aussi bien à la notion de groupe verbal, et il est d'ailleurs constamment rappelé que le prédicat correspond au GV dans la plupart des cas. Les divers avantages énumérés par les documents d'accompagnement des Programmes ("documents ressources») pourraient tout à fait être attribués au groupe verbal ; qu'il s'agisse « d'avoir une vision plus globale de la phrase et de sa structure en la décomposant en unités plus larges» (mais le prédicat n'est pas "plus large» que le GV), ou «d'envisager ensemble le verbe et ses éventuels compléments ", il n'y a pas là des propriétés qui seraient spécifiques au prédicat ; elles sont partagées par le groupe verbal, dont l'introduction dans la terminologie répondait d'ailleurs exactement à ces finalités didactiques. La superposition des deux notions apparait bien dans des formulations comme: «ramener une phrase complexe à une phrase simple, puisque l'une comme 
l'autre se composent d'un GN sujet et d'un GV prédicat » ou savoir identifier une phrase le GN sujet et le GV prédicat, c'est percevoir la « structure fondamentalement bipartite de la phrase canonique ", formulations où sont systématiquement associées les deux réalités dans l'expression « GV prédicat ».

Il faut se tourner vers une justification d'ordre plus théorique, que proposent les mêmes « documents-ressources ». Au plan de la description syntaxique, la notion de prédicat est destinée à combler un manque dans l'équilibre général des notions grammaticales. Il s'agit en effet de généraliser l'opposition nature/fonction et de ne pas la limiter aux constituants nominaux, adjectivaux ou adverbiaux, d'où le choix d'opposer le groupe verbal, en tant que classe, au prédicat, en tant que fonction. C'est ce que précisent les commentaires des textes officiels :

Parler de verbe ou de groupe verbal (GV), c'est indiquer une classe grammaticale, comme on le fait quand on parle de nom ou de groupe nominal (GN), de préposition ou de groupe prépositionnel, etc. - Parler de prédicat, c'est indiquer la fonction syntaxique du groupe verbal, comme on le fait quand on parle de sujet à propos d'un groupe nominal (GN). [...] C'est par souci de cohérence que le terme " prédicat » a été retenu pour nommer la fonction syntaxique du GV.

Même si, avec ce type d'argument, semble évité le danger du double emploi, il est sans doute toutefois un peu rapide d'estimer, comme cela est proposé dans cet extrait, que le prédicat est une "fonction" et non une nature de constituant, le parallèle avec les fonctions sujet ou complément étant bien trop simplificateur et réducteur. Alors que la relation «sujet »/prédicat, telle qu'elle est présentée, renvoie à l'opération de prédication, les relations qui s'établissent entre le verbe et ses compléments ne sont pas du même ordre. Le prédicat n'est pas dans une relation de dépendance par rapport à son sujet comme l'est un complément de verbe par rapport au verbe régissant. Parler de «fonction syntaxique» du groupe verbal comme on le ferait pour un circonstanciel ou pour une épithète masque des différences fondamentales et on rappellera d'ailleurs à ce sujet que la tradition n'a jamais reconnu au verbe une «fonction" grammaticale. En simplifiant trop, on aboutit à des impasses : lorsque le GV n'est pas prédicat, est-ce à dire qu'il n'a plus de "fonction syntaxique »? Cela soulève en particulier la délicate question des prédications "secondes ». Y aura-t-il prédication seconde sans prédicat? Dans: Remarquable, la réponse qu'il a faite! la subordonnée relative contient bien un GV. Faut-il le considérer comme un prédicat ou non? S'il n'a pas la fonction de prédicat, quelle fonction syntaxique lui attribuer? Ne doit-on reconnaitre comme prédicat que le prédicat principal? Bien sûr, on peut toujours revenir à la "phrase de base» ou phrase canonique : il a fait cette réponse et considérer qu'il y a là un prédicat qui perd son statut de prédicat principal dans l'opération de subordination, mais le groupe verbal de la relative n'a alors plus de fonction.

Se pose également la question $\mathrm{du}$ «sujet»: la clarification visée par la distinction prédicat/groupe verbal ne se retrouve pas lorsqu'il s'agit du sujet; ce dernier en effet correspond avant tout à une fonction syntaxique qui se définit par des propriétés dans une relation avec une forme verbale. Dans la mesure où le prédicat ne coïncide pas toujours avec le groupe verbal, une autre dénomination que sujet est nécessaire, sauf à reconnaitre deux types de sujet: le sujet du prédicat et le sujet du verbe. L'opposition classique thème/prédicat était attendue, mais il est vrai que la dénomination de "thème " est déjà utilisée pour décrire le niveau informationnel. Le maintien de l'étiquette "sujet» est évidemment contraire à la logique de clarification qui était annoncée. 
23 S'il s'agit d'adopter une terminologie qui accompagne de façon cohérente et opératoire une observation raisonnée des phénomènes linguistiques, la notion de prédication est assurément plus riche et plus utile que celle de prédicat. Une description de la langue qui essaie, comme cela est recommandé, de prendre en compte les caractéristiques sémantiques et pragmatiques peut difficilement faire l'économie de cette notion. $\mathrm{Si}$ l'opération de prédication et la valeur qu'elle représente sont bien comprises, la reconnaissance du prédicat, en tant que partie de la phrase, n'est plus un objectif prioritaire. Toutes proportions gardées, on se trouve dans le même cas de figure lorsqu'il s'agit de la transitivité, ou, plus largement, de la rection, et des compléments: l'observation et la réflexion ne peuvent se limiter à l'identification des unités mises en jeu, mais doivent avant tout porter sur le système de relations dans lequel entrent ces éléments et sur les opérations qui sous-tendent ce système.

\section{Conclusions} est pratiquée depuis déjà un certain nombre d'années; elles ne devraient pas permettre de faire l'économie de changements, beaucoup plus fondamentaux et plus profonds, qui essayeraient de mettre en accord les objectifs généraux et des propositions sur la description de la langue.

Pour ce qui est des contenus, sans délaisser la partie de la terminologie qui concerne les unités et les fonctions - les modifications proposées montrent qu'il y a encore des améliorations à apporter dans ce domaine -, il est urgent d'ajouter dans ce texte deux grandes parties, ce qui demanderait de procéder à deux types d'inventaire :

- Établir une nomenclature des structures linguistiques, des constructions, qui seront à aborder, et accompagner cette liste d'éléments de progression. Ceci implique de ne plus se satisfaire d'un programme de "grammaire » réduit en fait à un programme d'étiquetage grammatical, mais revient à élaborer ce qui a toujours été le grand absent des textes officiels : un véritable programme de langue.

- Énumérer et définir le plus clairement possible les termes désignant les opérations sousjacentes aux fonctions: détermination, caractérisation, prédication, rection, portée, modalisation, pour ne citer que quelques-unes d'entre elles.

Rappelons une nouvelle fois qu'il serait opportun, en ce qui concerne les finalités de la terminologie, de distinguer les notions destinées aux enseignants et les notions utilisées progressivement avec les élèves dans leur apprentissage.

Une fois clairement établies les notions renvoyant aux opérations et aux constructions, l'observation du système linguistique pourra s'effectuer sur de bonnes bases. C'est seulement alors, dans ce cadre général, que peut être éventuellement discutée la dénomination des éléments de l'énoncé, de leur nature et de leur fonction, question qui apparait finalement comme relativement secondaire.

MINISTÈRE DE L'ÉDUCATION NATIONALE, DE LA RECHERCHE ET DE LA TECHNOLOGIE (1997). Terminologie grammaticale. Paris : Centre national de documentation pédagogique. 


\section{BIBLIOGRAPHIE}

GENOUVRIER, É. \& PEYTARD, J. (1970). Linguistique et enseignement du français. Paris : Larousse.

MINISTÈRE DE L'ÉDUCATION NATIONALE, DE L'ENSEIGNEMENT SUPÉRIEUR ET DE LA RECHERCHE (2015). Bulletin

officiel spécial no 11 du 26 novembre 2015. Programmes d'enseignement du cycle des

apprentissages fondamentaux (cycle 2), du cycle de consolidation (cycle 3) et du cycle des

approfondissements (cycle 4). En ligne : http://cache.media.education.gouv.fr/file/

MEN_SPE_11/67/3/2015_programmes_cycles234_4_12_ok_508673.pdf.

\section{RÉSUMÉS}

Les modifications apportées à la terminologie grammaticale dans les derniers Programmes officiels sont replacées dans le cadre plus général des objectifs de l'enseignement de la langue. On soutient que la question de la terminologie des natures et des fonctions des éléments n'est pas prioritaire ; elle ne devrait être abordée qu'après l'élaboration d'une terminologie des structures de langue à étudier et des opérations qui sont sous-jacentes à ces structures.

The modifications brought to the grammatical terminology in the last official Programs are replaced in the more general frame of the objectives of language teaching. We support that the question of the terminology of the natures and the functions of constituents is not priority; it should be approached only after the elaboration of a terminology for structures of language to be studied and operations which are underlying in these structures.

\section{INDEX}

Mots-clés : terminologie grammaticale, programmes officiels, prédicat, prédication, proposition principale

Keywords : grammatical terminology, official syllabuses, predicate, predication, main phrase

\section{AUTEUR}

\section{BERNARD COMBETTES}

Université de Lorraine/Centre national de la recherche scientifique, ATILF, UMR 7118, F-54000, France 